УДК 681.3 .06

\title{
АЛГОРИТМ СИНТЕЗА ЭКОНОМИЧНЫХ СХЕМ З-БЛОКОВ ПОДСТАНОВКИ НА ОСНОВЕ КЛЕТОЧНЫХ АВТОМАТОВ
}

\author{
МАЗУРКОВ М. И., СОКОЛОВ А. В. \\ Одесский национальный политехнический университет, \\ Украина, Одесса, 65044, пр. Шевченко, 1
}

\begin{abstract}
Аннотация. Предложен алгоритм синтеза экономичных схем $S$-блоков подстановки на основе клеточных автоматов, которые удовлетворяют основным критериям криптографического качества. Найдены правила клеточных автоматов, позволяющие получить $S$-блоки подстановки, удовлетворяющие критерию максимального лавинного эффекта
\end{abstract}

Ключевые слова: клеточный автомат; S-блок подстановки; максимальный лавинный эффект

Вопросам конструирования криптографических биективных $S$-блоков подстановки, которые являются основными элементами современных блочных шифров, посвящено большое количество работ исследователей в области криптографии [1-3]. Современные методы конструирования высококачественных $S$-блоков подстановки подразумевают использование аппарата булевых функций для их описания [4]. Это позволяет достичь строго обоснованного уровня качества, которое определяется соответствием компонентных булевых функций конструируемого $S$-блока подстановки определенным критериям.

К таким критериям качества относятся высокая нелинейность, корреляционная независимость векторов выхода $S$-блока подстановки от его входа, строгий лавинный критерий, величина периодов возврата $S$-блока подстановки в исходное состояние. Однако, все чаще к требованиям конструируемых $S$-блоков подстановки относят критерий простоты их аппаратной или программной реализации [3]. Данное требование связано не только с концепци- ей энергоэффективности, но также с тем, что с ростом длины $S$-блока подстановки существенно улучшаются все его показатели криптографического качества [5]. Возможность реализовать $S$-блок подстановки большей длины при том же количестве аппаратных средств и энергоэффективности ведет к существенному улучшению характеристик криптоалгоритма, в котором такой $S$-блок подстановки применяется.

Так в случае использования криптографического $S$-блока подстановки, длина входного слова которого $k=32$ бита, потребуется хранить в памяти криптографической системы кодирующую $Q$-последовательность, определяющую структуру $S$-блока подстановки, длины $N=2^{k}=2^{32}=4294967296$. При этом, каждый элемент $Q$-последовательности представляет собой 32-разрядное число, т.е. необходимое для хранения $S$-блока подстановки количество памяти составит $4294967296 \times 32=$ $=137438953472$ бит $=16$ ГБ, что является весьма существенным объемом. При этом дальнейшее увеличение длины $S$-блока под- 